Journal of Emergency Primary Health Care An International elournal of Prehospital Care Research, Education, clinical Practice, Policy and Service Delivery ISSN 1447-4999

\title{
SPECIAL THEME - THROMBOLYSIS
}

Article 990154

\section{Early thrombolysis: time to change? A discussion paper}

\author{
Professor Malcolm Woollard \\ Faculty of Pre-hospital Care Research Unit, the James Cook University Hospital / University \\ of Teesside, Middlesbrough, UK.
}

\begin{abstract}
UK Department of Health standards for the management of out-of-hospital thrombolysis require a call to thrombolysis time of 60 minutes or less, but suggest that administration of such treatment in the pre-hospital setting should be limited to cases where the journey time to hospital exceeds 30 minutes. This policy was set despite more than $50 \%$ of patients in an urban setting having a call to hospital door time of more than 30 minutes, rising to more than $80 \%$ in rural areas, and that all published evidence suggests symptom to treatment time is the critical interval. Maximum benefits are derived from thrombolytic agents if they are delivered early. Administration within 30 minutes of symptom onset can result in total abortion of a myocardial infarction, and each minutes delay to treatment is equivalent to an average of 11 days of life lost. Pre-hospital lysis within two hours of symptom onset results in a significantly lower incidence of cardiogenic shock than percutaneous coronary intervention within the same time frame, suggesting greater salvage of cardiac muscle. The available evidence suggests that pre-hospital thrombolysis is at least as safe as in-hospital administration, regardless of the qualifications and experience of the practitioner providing the treatment. All patients benefit from the shortest possible interval from symptom onset to recanalization: minutes do count. UK standards should be amended to reflect this evidence and to mandate the administration of thrombolytic agents to all eligible patients as soon as they are identified in the pre-hospital setting, regardless of distance to hospital.
\end{abstract}

\section{Keywords}

emergency medical services; emergency medical technicians; myocardial infarction; prehospital; pre-hospital; thrombolytic therapy;

\section{Discussion}

In the UK, both the English and Welsh National Service Frameworks (NSF) for Coronary Heart Disease recommend a maximum call to thrombolysis time of 60 minutes for victims of myocardial infarction (MI). Pre-hospital thrombolysis is recommended to achieve this aim where the journey time to hospital exceeds 30 minutes. However, data from a large UK ambulance service with mixed topology indicates that cases with long call to hospital times are not limited to rural areas: 


\begin{tabular}{|l|l|l|l|}
\hline & $\begin{array}{l}\text { Urban area } \\
\text { (n= 3547) }\end{array}$ & $\begin{array}{l}\text { Rural area } \\
\text { (n= 526) }\end{array}$ & $\begin{array}{l}\text { Sparsely populated } \\
\text { area (n= 718) }\end{array}$ \\
\hline Mean (mins.) & 32.0 mins. & 46.6 mins. & 53.8 mins. \\
\hline Standard deviation & 10.0 mins. & 16.0 mins. & 23.7 mins. \\
\hline Range & 2.9 to 83.1 mins. & 8.1 to 97.8 mins & 6.7 to 136 mins. \\
\hline $\begin{array}{l}\text { Proportion of calls } \\
\text { exceeding call-to- } \\
\text { door time of 30 } \\
\text { minutes (\% of } \\
\text { calls, 95\% CI) }\end{array}$ & $\begin{array}{l}1886 / 3547 \text { (53.2\%, } \\
51.1 \text { to } 54.8 \%)\end{array}$ & $\begin{array}{l}438 / 526(83.3 \%, \\
79.8 \text { to } 86.4 \%)\end{array}$ & $\begin{array}{l}600 / 718(83.6 \%, \\
80.6 \text { to } 86.2 \%)\end{array}$ \\
\hline
\end{tabular}

Table 1: Call to hospital door times for chest pain patients in a large UK ambulance service with mixed topology (one year period)

There are a number of delays built in to the 999 call to hospital door interval (Box 1).

- 999 call processing

- Selection and activation of an ambulance

- Activation to mobilisation interval

- Journey time to scene

- Arrival on scene to arrival at the patient's side

- Provision of time-critical interventions on-scene

- Patient packaging

- Removal of patient from house

- Journey time to hospital

Box 1: Events resulting in delays in call to hospital times

Each of these intervals can be affected by a number of factors. Response times are influenced by the availability of responders and their location, themselves influenced by the workload of the ambulance service at any given time of day or day of the week. Traffic and other hazards will offer varying levels of impedance to the progress of the responders, and cannot, therefore, be regulated. There may be a delay in accessing the patient on arrival of responders due to their location. In urban areas, the patient may be on the top floor of an apartment block, and the lift may or may not be working. In rural areas they may be at the end of an unmade road that requires a walk of some distance from the ambulance. The patient will require some urgent interventions before being moved to the ambulance. These include the administration of oxygen, nitrates, aspirin, and opiates with an antiemetic - the latter drugs requiring the insertion of an intravenous cannula. Failure to correct pain risks increasing infarct size due to circulating catecholamines. Serious arrhythmias may require correction before the patient is moved, and if the 12-lead ECG is not recorded in the patient's home it is likely that the ambulance will be required to be stationary to obtain a trace of sufficient diagnostic quality. Many patients retire to bed when they feel unwell, and this slows the removal of the patient from the house. Journey times to hospital are influenced by the same factors as response time. Excessively rapid transportation will result in increased patient 
anxiety, increased catecholamine levels, and increased infarct size, and this effect will be exacerbated by the use of lights and sirens. In addition, if telemetry of ECG is used to prealert the receiving hospital, the ambulance may need to stop at a location where there is adequate signal strength to transmit the data.

It can be argued that, particularly where urgent percutaneous coronary intervention (PCI insertion of a stent) is unavailable, there is excellent evidence that thrombolytics should be administered in the pre-hospital phase of treatment in order to maximise patient benefit. Time is of the essence, and it is suggested that the NSF targets of a one-hour call to needle time should be regarded as a minimum level of achievement. The key to maximising benefit is minimising the time from the onset of major symptoms to administration of a thrombolytic. A one-hour call to treatment target ignores the considerable delays patients make in activating the emergency medical service. This delay in calling for help has been shown to be of the order of two hours. ${ }^{1}$

A meta-analysis of 22 randomised trials that compared thrombolytic therapy with placebo or controls showed that administration within one hour of symptom onset resulted in 65 lives saved per 1000 patients treated, but that the degree of benefit tailed off appreciably thereafter. $^{2}$ A further meta-analysis ${ }^{2}$ of these trials, but restricted to the relevant pre-hospital studies (including GREAT ${ }^{3}, \mathrm{EMIP}^{4}$, and $\mathrm{MITI}^{5}$ and with additional data from five smaller pre-hospital studies 678910 ), suggested an additional reduction in mortality at 35 days of 21 lives saved per 1000 patients, as a result of the further one hour saved on average (pain to thrombolysis time reduced from 3.1 to 2.1 hours). Using the same data, Rawles calculated that every minute of delay to thrombolytic therapy costs, on average, 11 days of life for patients treated in the first three hours following infarction. ${ }^{11}$ A more recent meta-analysis of six randomised controlled trials examining pre-hospital thrombolysis concluded that there is a decrease of $2 \%$ in the absolute risk of death per hour of time saved to treatment, and that prehospital thrombolysis saved an average of 45 minutes. ${ }^{12}$

Importantly, it has been shown that when reperfusion of an occluded coronary artery occurs within 15 to 30 minutes of the onset of symptoms, the prevention of any permanent damage to myocardium is possible. ${ }^{13}$ Unless pre-hospital thrombolysis eventually becomes routine for all appropriate patients, regardless of their proximity to a hospital, this benefit will never be realised other than for the few patients that infarct in hospital.

Safety issues are of considerable importance when considering the feasibility of pre-hospital thrombolysis. The MITI study, however, showed no increased risk of adverse events following pre-hospital administration of thrombolytic agents when compared to in-hospital therapy. ${ }^{5}$ This finding was confirmed by the meta-analysis of pre-hospital thrombolysis trials. ${ }^{12}$

The evidence concerning the relative value of early (pre-hospital) thrombolysis and PCI remains unclear. The Comparison of Angioplasty and Prehospital Thrombolysis In acute Myocardial infarction (CAPTIM) trial reported a strong (but not statistically significant) trend towards lower 30-day mortality in those patients randomised to receive pre-hospital thrombolysis within two hours of the onset of symptoms (2.2\% versus $5.7 \%, \mathrm{P}=0.058)$ but there was no between-groups difference in those entered into the study after an interval of greater than two hours. It also identified a significant reduction in the incidence of cardiogenic shock in those patients administered pre-hospital thrombolytics within two hours of symptom onset $(1.3 \%$ versus $5.3 \%, \mathrm{P}=0.032)$ but this relative benefit did not occur if treatment was delayed. ${ }^{14}$ It has been suggested that combining early pre-hospital thrombolysis with PCI may result in the greatest reduction in mortality, ${ }^{15}$ although evidence also exists to suggest that pre-hospital thrombolysis delivered within 3.5 hours is associated with a lower incidence of in-hospital death and a greater one year survival rate compared with in-hospital fibrinolysis and PCI. ${ }^{16}$ 
Approximately 10\% of 999 calls are to patients with chest pain, and a number are referred via a General Practitioner. 27\% of patients with an initial presentation of chest pain at the time of a 999 call for assistance will have suffered a myocardial infarction (Woollard M, Elwood P. Unpublished data). Of these, approximately half will ultimately be treated with thrombolytic drugs, and just under half of these of these may meet criteria for pre-hospital treatment. A delay of thirty minutes, inherent in any protocol that unnecessarily postpones drug administration until arrival at hospital, could cost each of these patients a year of life. It implies that all victims of out-of-hospital MI will suffer irreversible myocardial damage that may, in some patient, otherwise have been prevented. It is likely that some patients will consequently have a substantially reduced quality of life due to heart failure and this in turn has inevitable and considerable cost consequences for the NHS.

\section{Conclusion}

It is now an uncontested fact that the interval from onset of symptoms to recanalization of a coronary artery is a critical factor in predicting mortality and morbidity following myocardial infarction. Minutes really do count. The earlier reperfusion is facilitated, the greater the benefit, and if this can be initiated in less than 30 minutes some MIs can be aborted completely. For each patient presenting within three hours of the onset of symptoms, a delay of even thirty minutes could cost a year of life on average. There are a large number of factors that negatively influence call-to-door, and therefore call to in-hospital thrombolysis times. Even in an urban area, this delay exceeds 30 minutes in more than half of chest pain patients for whom a 999 call is made. And even when a facility offering emergent PCI is readily available, pre-hospital thrombolysis may offer a greater reduction in mortality and morbidity if administered within two to three hours. Perhaps it is time to amend the UK's targets for time to thrombolysis to reflect all of this the evidence and that symptom to pain time, not call to door time, is the critical interval. Relying on the latter can only result in poorer outcomes for patients. 


\section{References}

${ }^{1}$ Goff DC Jr, Feldman HA, McGovern PG, et al. Prehospital delay in patients hospitalized with heart attack symptoms in the United States: the REACT trial. Rapid Early Action for Coronary Treatment (REACT) Study Group. Am Heart J 1999;138(6 Pt 1):1046-57.

${ }^{2}$ Boersma E, Maas AC, Deckers JW, et al. Early thrombolytic treatment in acute myocardial infarction: reappraisal of the golden hour. Lancet 1996;348:771-5.

${ }^{3}$ GREAT Group. Feasibility, safety, and efficacy of domiciliary thrombolysis by general practitioners: Grampian region early anistreplase trial. BMJ 1992;305:548-53.

${ }^{4}$ The European Myocardial Infarction Project Group. Prehospital thrombolytic therapy in patients with suspected acute myocardial infarction. $N$ Engl J Med 1993;329:383-9.

${ }^{5}$ Weaver WD, Cerqueira M, Hallstrom AP, et al. Prehospital-initiated vs hospital-initiated thrombolytic therapy. The Myocardial Infarction Triage and Intervention Trial. JAMA 1993;270:1211-6.

${ }^{6}$ McNeill AJ, Cunningham SR, Flannery DJ, et al. A double blind placebo controlled study of early and late administration of recombinant tissue plasminogen activator in acute myocardial infarction. $\mathrm{Br}$ Heart J 1989;61:316-21.

${ }^{7}$ Schofer J, Buttner J, Geng G, et al. Prehospital thrombolysis in acute myocardial infarction. Am J Cardiol 1990;66:1429-33.

${ }^{8}$ Barbash GI, Roth A, Hod H, et al. Improved survival but not left ventricular function with early and prehospital treatment with tissue plasminogen activator in acute myocardial infarction. Am J Cardiol 1990;66:261-6.

${ }^{9}$ Castaigne AD, Herve C, Duval-Moulin AM, et al. Prehospital use of APSAC: results of a placebocontrolled study. Am J Cardiol 1989;64:30A-3A.

${ }^{10}$ McAleer B, Ruane B, Burke E, et al. Prehospital thrombolysis in a rural community: short- and longterm survival. Cardiovasc Drugs Ther 1992;6:369-72.

${ }^{11}$ Rawles JM. Quantification of the benefit of earlier thrombolytic therapy: five-year results of the Grampian Region Early Anistreplase Trial (GREAT). J Am Coll Cardiol 1997;30:1181-6.

12 Morrison LJ, Verbeek PR, McDonald AC, Sawadsky BV, Cook DJ. Mortality and Prehospital Thrombolysis for Acute Myocardial Infarction: A Meta Analysis. JAMA 2000;283(20):2686-2692.

${ }^{13}$ Saltissi S, Mushahwar SS. The management of acute myocardial infarction. Postgrad Med J 1995;71:534-41.

${ }^{14}$ Steg PG, Bonnefoy E, Chabaud S, Lapostolle F, Dubien PY, Cristofini P, Leizorovicz A, Touboul P; Comparison of Angioplasty and Prehospital Thrombolysis In acute Myocardial infarction (CAPTIM) Investigators. Impact of Time to Treatment on Mortality After Prehospital Fibrinolysis or Primary Angioplasty. Data From the CAPTIM Randomized Clinical Trial. Circulation 2003;108(23):2851-6.

${ }^{15}$ Smalling RW, Giesler G. The level I cardiovascular center: is it time? Am Heart Hosp J 2003;1(2):170-4.

${ }^{16}$ Danchin N, Blanchard D, Steg PG, Sauval P, Hanania G, Goldstein P, Cambou JP, Gueret P, Vaur L, Boutalbi Y, Genes N, Lablanche JM; USIC 2000 Investigators. Impact of prehospital thrombolysis for acute myocardial infarction on 1-year outcome: results from the French Nationwide USIC 2000 Registry. Circulation 2004;110(14):1909-15.

This Article was peer reviewed for the Journal of Emergency Primary Health Care Vol.3, Issue 4, 2005 